\title{
Les élites maliennes en quête d'Amérique : Migrations internationales pour études et enjeux d'une reproduction sociale inachevée
}

Thèse soutenue le 21 novembre 2014 à l'université de Caen Normandie, en cotutelle avec l'université de Poitiers

\section{Kévin Mary}

\section{CpenEdition}

\section{Journals}

Édition électronique

URL : https://journals.openedition.org/e-migrinter/688

DOI : 10.4000/e-migrinter.688

ISSN : 1961-9685

Éditeur

UMR 7301 - Migrinter

Référence électronique

Kévin Mary, «Les élites maliennes en quête d'Amérique : Migrations internationales pour études et enjeux d'une reproduction sociale inachevée », e-Migrinter [En ligne], 14 | 2016, mis en ligne le , consulté le 20 mai 2021. URL : http://journals.openedition.org/e-migrinter/688 ; DOI : https://doi.org/ 10.4000/e-migrinter.688

Ce document a été généré automatiquement le 20 mai 2021.

Tous droits réservés 


\section{Les élites maliennes en quête d'Amérique : Migrations internationales pour études et enjeux d'une reproduction sociale inachevée}

Thèse soutenue le 21 novembre 2014 à l'université de Caen Normandie, en cotutelle avec l'université de Poitiers

\section{Kévin Mary}

\section{RÉFÉRENCE}

Mary, Kévin (2014) Les élites maliennes en quête d'Amérique : Migrations internationales pour études et enjeux d'une reproduction sociale inachevée, Caen, Université de Caen. Soutenance de thèse.

1 La crise qui a touché le Mali en 2012 et 2013 a mis en exergue un certain nombre de fragilités propres à la société malienne. Les analyses de ces soubresauts ont souvent pointé du doigt, parmi d'autres facteurs, la responsabilité des élites en place dans le délitement du pays. Cette thèse, dans la mesure où l'étude des élites signifie ici d'entreprendre une analyse " par le haut » des inégalités sociales (Khan, 2012, p. 362), aspire, par son objet, à contribuer à la compréhension de quelques-uns des éléments qui ont pu mener à une telle situation.

2 Centrée plus précisément sur les trajectoires migratoires des enfants issus des familles d'hommes politiques, de grands commerçants ou de hauts fonctionnaires maliens, la démarche de ce travail de thèse consiste en un croisement entre la géographie sociale et la géographie des migrations. Elle met en relief un espace migratoire délimité, puisqu'elle s'intéresse aux migrations pour études des enfants d'élites maliennes vers 
les États-Unis et le Canada, mais pointe, au delà de présenter le pays de départ et ceux d'arrivée, un continuum migratoire produit par les élites. En effet, cette recherche s'intéresse aux conditions de reproduction d'un groupe social par la mobilité. La place des migrations pour études dans la formation de la classe des élites s'avère ainsi centrale dans l'analyse : les jeunes maliens issus de familles aisées partent-ils étudier en Amérique du Nord pour ne pas revenir, ou pour «mieux revenir » et afficher leurs appartenances à la catégorie des élites?

3 La thèse suit donc le parcours de ces jeunes maliens, depuis leur volonté d'aller étudier aux États-Unis ou au Canada, jusqu'à leur éventuel retour au Mali à l'issue de leurs études supérieures.

4 À Bamako, une enquête par questionnaire $(\mathrm{N}=940)$ a été menée auprès d'élèves de plusieurs lycées aisés de la capitale malienne dans le but de mesurer l'attrait pour les études à l'étranger et d'en saisir certaines des logiques. Cette enquête a d'abord permis de montrer que dans les familles d'élites, la poursuite d'études au Mali n'est pas envisageable et l'investissement dans le «label» étranger, réputé plus sûr et plus sérieux, quasiment systématique. Cela confirme le manque de confiance - pour les familles qui en ont les moyens - dans le système d'enseignement supérieur malien, qui connait depuis les années 1980 une crise endémique. Mais au delà de cet aspect, l'enquête montre que la pratique des études à l'étranger relève également de véritables « désirs d'ailleurs » qui s'inscrivent chez les élites dans la suite logique de modes de vie tournés vers l'extérieur - des modes de vies « extravertis » dirait J-F. Bayart (1989) dans lesquels les voyages occupent une place importante. Un troisième enseignement de l'enquête par questionnaire réside dans la dénomination des pays d'études plébiscité par la «jeunesse dorée " malienne. À cet égard, l'Amérique du Nord fait visiblement rêver, peut-être aujourd'hui plus qu'une destination "historique » comme la France dont la relation complexe avec le Mali semble aujourd'hui relever d'une dynamique de «proximité sans réciprocité », pour reprendre l'expression d'A. Mbembe (2013). Mais on touche aussi ici à la problématique de l'internationalisation du champ de l'éducation supérieure et de la concurrence que se livrent les territoires dans le cadre de ce que l'on nomme parfois "l'économie du savoir». Dans ce contexte, les universités américaines et canadiennes tirent partie de politiques volontaristes d'attraction d'étudiants étrangers, y compris Africains. Le cas de l'université de Moncton au Canada est plus spécifiquement développé et donne à voir cette stratégie appliquée à l'accueil des étudiants maliens les plus solvables. Plusieurs logiques se combinent donc ici. L'une plutôt « répulsive » qui incite ceux ayant les moyens à aller étudier ailleurs qu'au Mali au regard de la situation de l'enseignement supérieur local, les autres plutôt « attractives » qui associent des modes de vies de type « extravertis » et des politiques incitatives d'accueil d'étudiants africains de la part des universités nord-américaines.

Une série d'entretiens a ensuite été menée auprès d'une vingtaine de lycéens bamakois ayant participé à l'enquête par questionnaire. Ces entretiens ont permis de mettre à jour des liens d'interconnaissance forts entre les familles membres de l'élite. Par l'intermédiaire notamment de mariages ou encore de scolarisation dans les mêmes établissements (où enfants d'hommes politiques et de grands commerçants, pourtant issus de mondes sociaux bien distincts, se côtoient dès l'adolescence) ces familles montrent leur souci de se créer un entre-soi. Cet entre-soi permet en effet de bénéficier d'un important capital relationnel, appelé mògòtigiya, en bambara ${ }^{1}$. Là où l'enquête par questionnaire pointait l'important capital économique détenu pas les familles d'élites 
maliennes, les entretiens ont fait ressortir leur capital social. Ces observations renvoient ainsi à celles de R. Vuarin (1994) pour qui "l'argent et l'entregent" constituent la dialectique principale des processus d'accumulation au Mali et représentent les attributs sociaux qui ont trait à la domination.

Forts de cette "puissance sociale ", une partie des enfants de la bourgeoisie malienne part étudier en Amérique du Nord. Des terrains aux États-Unis (Washington DC et New York) et au Canada (Moncton) ont été investis sur un mode qualitatif et des entretiens ont été conduits avec des étudiants maliens dans ces deux pays. Cette démarche a fait émerger plusieurs résultats. Elle montre d'abord un ajustement long et difficile aux études en langue anglaise, qui occupe les premières années universitaires des étudiants maliens. Le passage d'un environnement francophone à un environnement anglophone ne va en effet pas de soi : il nécessite temps et argent. Car le coût élevé des études en Amérique du Nord constitue une barrière importante, et beaucoup de parcours d'étudiants se voient en fait détournés. Cette barrière effectue un tri parmi les différentes fractions de l'élite, et seuls les plus privilégiés arrivent au bout de leurs études et décrochent des diplômes. D'autre part, le choix des filières d'études s'est révélé assez homogène. Les "enfants des riches" se destinent dans leur majorité à devenir de futurs "gestionnaires » et plébiscitent les diplômes en administration des Affaires. En revanche, l'institution qui délivre ces diplômes compte peu semble-t-il dans le choix des étudiants maliens, à l'image de l'université de Moncton qui est une université périphérique au Canada (Wade et Belkhodja, 2011). En d'autres termes, cela signifie qu'ils ne s'inscrivent généralement ni dans les universités nord-américaines les plus prestigieuses ni les plus chères, à quelques exceptions près. Le système d'enseignement supérieur nord-américain se révèle en effet relativement méconnu des étudiants maliens.

7 Lorsqu'une partie de ces étudiants - et bien qu'il s'avère impossible d'en donner une estimation précise ${ }^{2}$-, munis de leurs diplômes nord-américains, retourne au Mali, leur statut social semble cependant être marqué par certaines formes de fragilités. La méthode d'investigation est ici basée sur le recueil de récits d'histoires familiales et d'observations menées à Bamako, au cours d'un dernier terrain. Il permet d'affiner l'analyse en portant un regard sur la structure interne de l'élite et de ses reconfigurations.

8 Ce retour constitue en un certain sens une réussite puisque ces diplômés conservent des positions privilégiées. Munis de diplômes en administrations des Affaires et animés par un idéal de la figure de l'entrepreneur privé, les « enfants des riches » parviennent à trouver un emploi salarié, souvent dans les entreprises du secteur privé: ils deviennent cadres dans les banques ou dans les entreprises de télécommunications présentes au Mali. Ils sont par contre moins nombreux à envisager d'intégrer la fonction publique, alors même que les itinéraires d'accumulation les plus efficaces résident dans l'accès à l'État et aux postes qui lui sont liés.

9 Mais les diplômes en administration des Affaires décernés par les universités nordaméricaines se négocient de manière plus ambiguë dans la sphère de l'État. Pourtant, c'est depuis cette sphère que la majeure partie de la rente est redistribuée. L'accès aux postes de la haute administration publique est donc primordial pour pouvoir «tenir son rang " au sein de l'élite. Cela montre qu'au Mali la reproduction sociale est avant tout celle de la reproduction du système politique. Et le capital culturel que constituent ces diplômes ne s'avère pas suffisant pour intégrer un État dont les réseaux opèrent sur 
des bases clientélistes. La dynamique des réseaux de pouvoir rappelle que l'État doit être pensé comme "la condensation d'un rapport de force " entre les différents courants qui le traverse, pour reprendre une formulation du philosophe Nikos Poulantzas (1978), où de nouvelles figures de la réussite sont susceptibles d'émerger, à l'image par exemple de l'arrivée en force de l'islam politique et de certains de ses leaders (Holder, 2013). Cette configuration ne garantie pas une reproduction automatique du statut social des familles d'élites, et montre au contraire que la domination peut rencontrer des « complications » (Hibou, 2011).

Ainsi, l'un des apports de la thèse demeure dans son ambition d'interroger les luttes qui se jouent à l'intérieur de l'élite. Cela permet d'éviter de donner une vision univoque de la domination qui verrait la genèse claire d'un groupe sur un autre. En montrant le déclassement possible d'élites même placées au sommet de la hiérarchie sociale, ce travail montre que la reproduction demeure, au moins pour une part, inachevée.

\section{BIBLIOGRAPHIE}

Bayart, Jean-François (1989) L'État en Afrique, Paris, Fayard, 439 p.

Hibou, Béatrice (2011) Anatomie politique de la domination, Paris, La Découverte, 298 p.

Holder, Gilles (2013) Un pays musulman en quête d'État-nation, in Gonin, P. ; Kotlok, N. ; Pérouse de Montclos, A. (dir.) La tragédie malienne, Paris, Vendémiaire, pp. 131-157.

Khan, Shamus (2012) Sociology of elites, Annual Review of Sociology, n³8, pp. 361-377.

Mbembe, Achille (2013) Sortir de la grande nuit, Paris, La Découverte, 254 p.

Poulantzas, Nicos (1978) L'État, le pouvoir, le socialisme, Paris, PUF, $300 \mathrm{p}$.

Vuarin, Robert (1993) L'argent et l'entregent, Cahiers des Sciences humaines, vol. 30, n 1-2, pp. 255-273.

Wade, Mathieu ; Belkhodja, Chedly (2011) Managing a new diversity in small campuses, Diversité canadienne, vol. 8, n 5, pp. 39-42.

\section{NOTES}

1. De « mògò ", personne, homme, et "tigiya », possession, détention. Littéralement, celui qui a des gens, qui possède des hommes.

2. Même s'il est néanmoins possible d'affirmer que le retour dans le pays d'origine à l'issue des études n'est pas un phénomène rare ou isolé dans ces milieux sociaux. 
INDEX

Index géographique : Mali, États-Unis

Mots-clés : étudiants, mobilité géographique, inégalité sociale, relations avec le pays d'origine, thèse, Maliens

\section{AUTEURS}

\section{KÉVIN MARY}

Maître de conférences en géographie, ART-Dev - UMR 5281 CNRS, Université de Perpignan Via Domitia

kevin.mary@univ-perp.fr 